Title:

A computerized training program for teaching people how to plan better

Authors:

Falk Lieder*, Paul M. Krueger*, Frederick Callaway*, Thomas L. Griffiths

* These authors contributed equally.

\title{
100-word abstract
}

We present an intelligent tutoring system for teaching people effective planning strategies. Our training program combines a novel process-tracing paradigm that makes people's latent planning strategies observable with an AI systems that gives people immediate feedback on how close their planning strategy is to optimal planning. Three experiments demonstrate that our method can automatically discover which planning strategies is optimal for a given class of problems and teach it to people. We find that the metacognitive process feedback provided by our method accelerates learning compared to no-feedback and conventional feedback on the quality of the selected actions, and the training effects are retained after a break even when the feedback is removed.

\section{0-word abstract:}

While some people learn to make excellent decisions by planning far ahead others learn to rely on short-sighted heuristics. Recent work suggests that the mechanisms by which people learn how to decide can be understood in terms of metacognitive reinforcement learning (Krueger, Lieder, \& Griffiths, 2017). Here, we leverage this perspective to develop a computerized cognitive training program that teaches people to make better decisions by giving them immediate feedback on the quality of their planning process.

To develop this training paradigm, we made four contributions to the literatures on decisionmaking, bounded rationality, and cognitive training: i) a normative theory of planning under limited time and bounded cognitive resources that can be used to quantify the quality of alternative planning operations, ii) a computational model of how people learn how to decide, iii) a process tracing paradigm that makes the latent cognitive process of planning observable, and iv) a computational method for computing optimal metacognitive feedback signals and messages.

The basic idea of our normative theory is that people should make optimal use of their finite time and limited cognitive resources. Our model of metacognitive learning postulates that our cognitive processes are shaped by the reinforcement learning mechanisms that are very similar to those that shape our habits. Our new Mouselab-MDP paradigm traces how people plan by requiring them to click through the sequence of states they are considering to visit to find out how much money they would yield. This allows us to operationalize planning operations by where people click. This allows us to compute optimal feedback on the planning process by 
comparing the value of the planning operation selected by the participant to the value of the optimal planning operation. We determine the value of planning operations according to the theory of rational metareasoning developed in the artificial intelligence literature (Russell \& Wefald, 1991). Intuitively, the value of a planning operation is defined as the expected improvement in decision quality minus its opportunity cost.

We report a series of controlled experiments showing that our method can effectively accelerate how people learn to plan better. In Experiment 1 we found that our method can automatically discover which planning strategy is optimal for a given environment and deliver optimal feedback for training people to adopt it. Participants receiving this feedback learned significantly faster and achieve a higher level of performance. In Experiment 2 we found using our method to give people feedback on their planning process was significantly more effective than giving people feedback on their decisions directly. Finally, Experiment 3 demonstrated that the benefits of training people with our method are retained when the feedback is removed and still persist after a $10 \mathrm{~min}$ break.

These findings inspire optimism that our account of bounded rationality and metacognitive learning might provide a principled theoretical foundation for cognitive training and enable more effective interventions for improving the human mind by giving feedback that is optimized for promoting metacognitive reinforcement learning. We hope that our optimal feedback will prove useful for cognitive training of higher cognitive functions such as planning, decision making, and problem solving.

References:

1. Krueger, P.M.*, Lieder, F.*, Griffiths, T.L. (2017). Enhancing Metacognitive Reinforcement learning using reward structures and feedback. Proceedings of the 39th Annual Meeting of the Cognitive Science Society.

2. Callaway, F.* Lieder, F.*, \& Krueger, P.M.*, \& Griffiths, T.L. (2017). Mouselab-MDP: A new paradigm for tracing how people plan. The 3rd Multidisciplinary Conference on Reinforcement Learning and Decision Making.

3. Russell, S., \& Wefald, E. (1991). Principles of metareasoning. Artificial intelligence, 49(1-3), 361-395. 